\title{
Operational matrix approach for the nonlinear Volterra- Fredholm integral equations: Arising in physics and engineering
}

\author{
B. Basirat ${ }^{1 *}, \mathrm{~K}$. Maleknejad $^{2}$ and E. Hashemizadeh ${ }^{2}$ \\ ${ }^{1}$ Department of Mathematics, Birjand Branch, Islamic Azad University, Birjand, Iran. \\ ${ }^{2}$ Department of Mathematics, Karaj Branch, Islamic Azad University, Karaj, Iran.
}

Accepted 28 December, 2011

\begin{abstract}
An approximation method based on hybrid Legendre polynomials and Block-Pulse functions used for the solution of nonlinear Volterra-Fredholm integral equations (NV-FIEs). The operational matrices of these functions are utilized to reduce a nonlinear Volterra-Fredholm integral equation to a system of nonlinear algebraic equations. In addition, convergence analysis and numerical examples that illustrate the pertinent features of the method are presented.
\end{abstract}

Key words: Hybrid functions, nonlinear integral equations, operational matrix, product matrix, coefficient matrix.

\section{INTRODUCTION}

Integral equation has been one of the principal tools in various areas of applied mathematics, physics and engineering. Integral equation is encountered in a variety of applications in many fields, including continuum mechanics, potential theory, geophysics, electricity and magnetism, antenna synthesis problem, communication theory, mathematical economics, population genetics and radiation, the particle transport problems of astrophysics and reactor theory, fluid mechanics etc. Many of these integral equations are nonlinear (Voitovich and Reshnyak, 1999; Jaswon and Symm, 1977; Schiavane et al., 2002; Abdou, 2003; Bloom, 1980; Jiang and Rokhlin, 2004; Semetanian, 2007).

In this paper we deal with nonlinear Volterra-Fredholm integral equations as follows:

$$
u(x)=f(x)+\lambda_{1} \int_{0}^{x} k_{1}(x, s) \psi_{1}(s, u(s)) d s+\lambda_{2} \int_{0}^{1} k_{2}(x, s) \psi_{2}(s, u(s)) d s,
$$

where the parameters $\lambda_{1}, \lambda_{2}$ and

*Corresponding author. E-mail: behrooz.basirat@kiau.ac.ir.

Mathematics subject classification (2010): 45G10, 45D05, 45B05, 40C05. functions $f(x), \psi_{1}(s, u(s)), \quad \psi_{2}(s, u(s)), \quad k_{1}(x, s)$ and $k_{2}(x, s)$ are known while $L^{2}[0,1)$ and $u(x)$ is an unknown function. In this work we suppose $\psi_{1}(s, u(s))=u(s)^{\alpha} \quad$ and $\quad \psi_{2}(s, u(s))=u(s)^{\beta} \quad$ where $\alpha, \beta$ are positive integers (Maleknejad et al., 2011; Maleknejad et al., 2010; Yalcinbas, 2002; Yousefi and Razzaghi, 2005; Ordokhani, 2006; Babolian et al., 2008; Sepehrian and Razzaghi, 2005; Ordokhani and Razzaghi, 2008)

Maleknejad, Hashemizadeh and Basirat solved Equation 1 by Bernstein operational matrices method (Maleknejad et al., 2011). Maleknejad, Almasih and Roodaki reduced these kinds of equations to algebraic equations by triangular functions (Maleknejad et al., 2010). Yalcinbas (2002) applied Taylor series to solve Equation 1. Yousefi and Razzaghi (2005) used Legendre wavelets for the numerical solution of these equations. Ordokhani (2006) used rationalized Haar functions for solving these equations and Babolian et al. (2008) used Block-Pulse functions to encounter these kinds of NVFIEs.

In this paper we use the hybrid Legendre polynomials and Block-Pulse functions as basis for reducing NV-FIEs to a system of nonlinear algebraic equations. We present hybrid Legendre polynomials and Block-Pulse useful 
properties such as operational matrix of integration, product matrix, integration of the cross product and coefficient matrix, and use them to transform our NV-FIE to an algebraic system. As shown in our examples, our method works better in comparison to the existing methods.

This paper introduces hybrid functions and their properties. Application of these set of hybrid functions for approximating the solution of NV-FIEs was done, convergence analysis given and the proposed method tested with some examples; thereafter the results were compared with some existing methods results. Thus a conclusion was drawn.

\section{HYBRID FUNCTIONS OF BLOCK-PULSE AND LEGENDRE POLYNOMIALS}

The orthogonal set of hybrid functions is $h_{i j}(x), i=1,2, \ldots, n, j=0,1, \ldots, m-1$ in which $i$ is the order for Block-Pulse functions, $j$ is the order for Legendre polynomials, and $X$ is the normalized time and is defined on the interval $[0,1)$ (Hsiao, 2009).

$$
h_{i j}(x)= \begin{cases}L_{j}(2 n x-2 i+1), & \frac{i-1}{n} \leq x<\frac{i}{n}, \\ 0, & \text { otherwise. }\end{cases}
$$

Here, the Legendre polynomials $L_{m}(x)$ on the interval $^{[-1,1]}$ :

$$
\begin{aligned}
& L_{0}(x)=1, L_{1}(x)=x, \\
& (m+1) L_{m+1}(x)=(2 m+1) x L_{m}(x)-m L_{m-1}(x), m=1,2,3, \ldots .
\end{aligned}
$$

The set $\left\{L_{m}(x): m=0,1, \ldots\right\}$ in Hilbert space $L^{2}[-1,1]$ is a complete orthogonal set.

A set of Block-Pulse functions $b_{i}(x), i=1,2, . ., n$ on the interval $^{[0,1)}$ is defined as follows:

$$
b_{i}(x)= \begin{cases}1, & \frac{i-1}{n} \leq x<\frac{i}{n} \\ 0, & \text { otherwise }\end{cases}
$$

The Block-Pulse functions on $[0,1)$ are disjoint, that results for $i, j=1,2, \ldots, n$, we have: $b_{i}(x) b_{j}(x)=\delta_{i j} b_{i}(x)$, also these functions have the property of orthogonality on $[0,1)$. Since $h_{i j}(x)$ is the combination of Legendre polynomials and Block-Pulse functions which are both complete and orthogonal, thus the set of hybrid functions is complete orthogonal set too.

\section{PROPERTIES OF HYBRID FUNCTIONS}

\section{Function approximation}

Any function $u(x) \in L^{2}[0,1)$ can be expanded in hybrid functions (Hsiao, 2009).

$$
u(x)=\sum_{i=1}^{\infty} \sum_{j=0}^{\infty} c_{i j} h_{i j}(x)
$$

where the hybrid coefficients are given by $c_{i j}=\frac{\left(u(x), h_{i j}(x)\right)}{\left(h_{i j}(x), h_{i j}(x)\right)}$ for $i=1,2, \ldots, \infty, j=0,1, \ldots, \infty$, so that, $(\cdot, \cdot)$ denotes the inner product.

Usually, the series expansion Equation 4 contains an infinite number of terms for a smooth $u(x)$. If $u(x)$ is piecewise constant or may be approximated as piecewise constant, then the sum in Equation4 may be terminated after $n m$ terms, that is,

$$
u(x) \simeq \sum_{i=1}^{n} \sum_{j=0}^{m-1} c_{i j} h_{i j}(x)=C^{T} \mathbf{h}(x)
$$

where

$$
C=\left[c_{10}, \ldots, C_{1, m-1}, C_{20}, \ldots, C_{2, m-1}, \ldots, C_{n 0}, \ldots, C_{n, m-1}\right]^{T}
$$

$$
\mathbf{h}(x)=\left[h_{10}(x), \ldots, h_{1 m-1}(x), h_{20}(x), \ldots, h_{2 m-1}(x), \ldots, h_{m-1}(x)\right]^{T}
$$

We can also approximate the function $k(x, s) \in L^{2}([0,1) \times[0,1))$ as follows:

$$
k(x, s) \simeq \mathbf{h}^{T}(x) K \mathbf{h}(s),
$$

where $K$ is an $n m \times n m$ matrix that $K_{i j}=\frac{\left(\mathbf{h}_{(i)}(x),\left(k(x, s), \mathbf{h}_{(j)}(s)\right)\right)}{\left(\mathbf{h}_{(i)}(x), \mathbf{h}_{(i)}(x)\right)\left(\mathbf{h}_{(j)}(s), \mathbf{h}_{(j)}(s)\right)}$ for $i, j=1,2, \ldots, n m$

\section{Operational matrix of integration}

The integration of the vector $\mathbf{h}(x)$ defined in Equation 7 
is given by

$$
\int_{0}^{x} \mathbf{h}\left(x^{\prime}\right) d x^{\prime} \simeq P \mathbf{h}(x)
$$

where $P$ is the $n m \times n m$ operational matrix for integration and is given (Hsiao, 2009; Chang and Wang, 1983) as:

$P=\left[\begin{array}{ccccc}E & H & H & \ldots & H \\ O & E & H & \ldots & H \\ O & O & E & \ldots & H \\ \vdots & \vdots & \vdots & \ddots & \vdots \\ O & O & O & \ldots & E\end{array}\right]$,

that $E$ and $H$ are $m \times m$ matrices that have the following shapes, respectively.

$$
\begin{aligned}
& H=\frac{1}{n}\left[\begin{array}{ccccc}
1 & 0 & 0 & \cdots & 0 \\
0 & 0 & 0 & \cdots & 0 \\
0 & 0 & 0 & \cdots & 0 \\
\vdots & \vdots & \vdots & \ddots & \vdots \\
0 & 0 & 0 & \cdots & 0
\end{array}\right], \\
& E=\frac{1}{2 n}\left[\begin{array}{ccccccccccc}
1 & 1 & 0 & 0 & 0 & \cdots & 0 & 0 & 0 & 0 & 0 \\
\frac{-1}{3} & 0 & \frac{1}{3} & 0 & 0 & \cdots & 0 & 0 & 0 & 0 & 0 \\
0 & \frac{-1}{5} & 0 & \frac{1}{5} & 0 & \cdots & 0 & 0 & 0 & 0 & 0 \\
0 & 0 & \frac{-1}{7} & 0 & \frac{1}{7} & \vdots & 0 & 0 & 0 & 0 & 0 \\
0 & 0 & 0 & \frac{-1}{9} & 0 & \cdots & 0 & 0 & 0 & 0 & 0 \\
\vdots & \vdots & \vdots & \vdots & \vdots & \ddots & \vdots & \vdots & \vdots & \vdots & \vdots \\
0 & 0 & 0 & 0 & 0 & \cdots & 0 & \frac{1}{2 m-9} & 0 & 0 & 0 \\
0 & 0 & 0 & 0 & 0 & \cdots & \frac{-1}{2 m-7} & 0 & \frac{1}{2 m-7} & 0 & 0 \\
0 & 0 & 0 & 0 & 0 & \cdots & 0 & \frac{-1}{2 m-5} & 0 & \frac{1}{2 m-5} & 0 \\
0 & 0 & 0 & 0 & 0 & \cdots & 0 & 0 & \frac{-1}{2 m-3} & 0 & \frac{1}{2 m-3} \\
0 & 0 & 0 & 0 & 0 & \cdots & 0 & 0 & 0 & \frac{-1}{2 m-1} & 0
\end{array}\right] .
\end{aligned}
$$

\section{The integration of the cross product}

The integration of the cross product of two hybrid function vectors $\mathbf{h}(x)$ in Equation 7 can be obtained as

$$
D=\int_{0}^{1} \mathbf{h}(x) \mathbf{h}^{T}(x) d x=\left[\begin{array}{cccc}
L & O & \ldots & O \\
O & L & \ldots & O \\
\vdots & \vdots & \ddots & \vdots \\
O & O & \ldots & L
\end{array}\right],
$$

where $L$ is an $m \times m$ diagonal matrix that is given by

$$
L=\frac{1}{n}\left[\begin{array}{cccc}
1 & 0 & \ldots & 0 \\
0 & \frac{1}{3} & \ldots & 0 \\
\vdots & \vdots & \ddots & \vdots \\
0 & 0 & \ldots & \frac{1}{2 m-1}
\end{array}\right] .
$$

The efficacy of matrix $D$ is used for converting the Fredholm part of NV-FIEs to an algebraic equation. Because of its diagonal shape, it can increase the calculating speed.

\section{Product operational matrix}

It is always necessary to evaluate the product of $\mathbf{h}(x)$ and $\mathbf{h}^{T}(x)$, that is called the product matrix of hybrid functions. Let

$$
\mathbf{H}(x)=\mathbf{h}(x) \mathbf{h}^{T}(x),
$$

where $\mathbf{H}(x)$ is an $n m \times n m$ matrix. Multiplying the matrix $\mathbf{H}(x)$ in vector $C$ that was defined in Equation 6, we obtain

$$
\mathbf{H}(x) C=\tilde{C} \mathbf{h}(x),
$$

where $\tilde{C}$ is an $n m \times n m$ matrix and is called the coefficient matrix. To illustrate the calculation procedure in Equation 13 we consider that $n=4$ and $m=3$ (Hsiao, 2009; Chang and Wang, 1983; Marzban and Razzaghi, 2003). We have

$h_{i j}(x) h_{k l}(x)=0$ if $i \neq k$

$h_{i 0}(x) h_{i j}(x)=h_{i j}(x)$

$h_{i 1}(x) h_{i 1}(x)=\frac{1}{3} h_{i 0}(x)+\frac{2}{3} h_{i 2}(x)$

$h_{i 1}(x) h_{i 2}(x)=\frac{2}{5} h_{i 1}(x)+\frac{3}{5} h_{i 3}(x)$

$h_{i 2}(x) h_{i 2}(x)=\frac{1}{5} h_{i 0}(x)+\frac{2}{7} h_{i 2}(x)+\frac{18}{35} h_{i 4}(x)$. 
Then we get

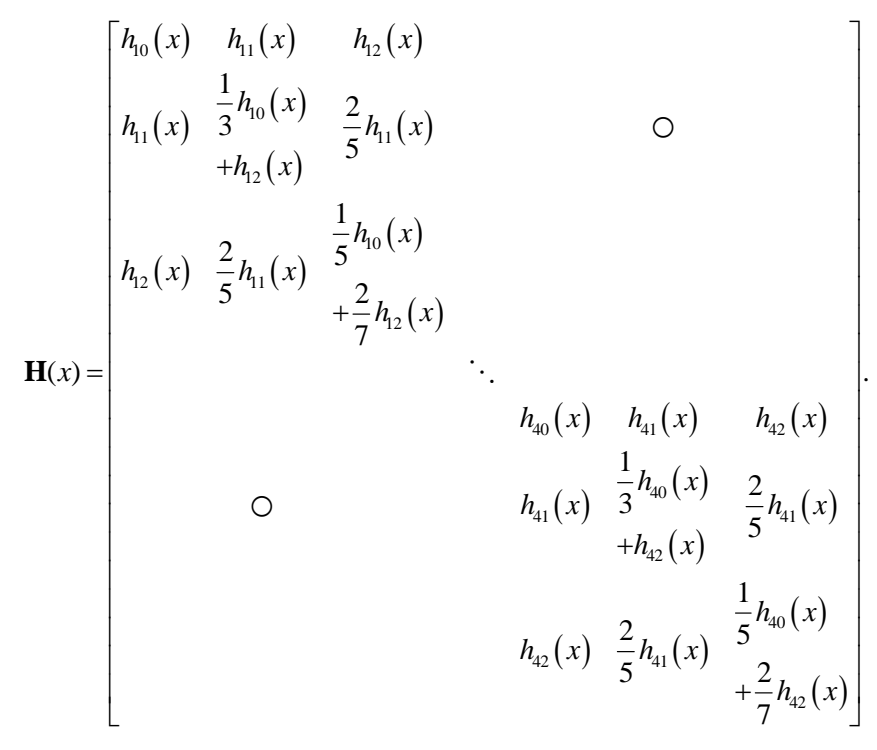

The matrix $\tilde{C}_{12 \times 12}$ in Equation 13 is given by

$$
\tilde{C}=\left[\begin{array}{cccc}
\widetilde{C_{1}} & O & O & O \\
O & \widetilde{C_{2}} & O & O \\
O & O & \widetilde{C_{3}} & O \\
O & O & O & \widetilde{C}_{4}
\end{array}\right],
$$

where $\widetilde{C}_{i}, i=1, \ldots, 4$ are given by

$$
\widetilde{C}_{i}=\left[\begin{array}{ccc}
c_{i 0} & c_{i 1} & c_{i 2} \\
\frac{1}{3} c_{i 1} & c_{i 0}+\frac{2}{5} c_{i 2} & \frac{2}{3} c_{i 1} \\
\frac{1}{5} c_{i 2} & \frac{2}{5} c_{i 1} & c_{i 0}+\frac{2}{7} c_{i 2}
\end{array}\right] .
$$

With the powerful properties of Equation 13, we can convert the Volterra part of NV-FIEs to an algebraic equation.

\section{OUTLINE OF THE METHOD FOR NV-FIES VIA HYBRID FUNCTIONS}

Consider the nonlinear Volterra-Fredholm integral Equation 1. We put

$$
u(x) \simeq U^{T} \mathbf{h}(x),
$$

where $U$ is an unknown $n m_{\text {-vector and }} \mathbf{h}(x)$ is given by Equation 7.

Likewise $k_{1}(x, s), k_{2}(x, s)$ and $f(x)$ are expanded into the hybrid functions as follows:

$$
\begin{aligned}
& k_{1}(x, s) \simeq \mathbf{h}^{T}(x) K_{1} \mathbf{h}(s), k_{2}(x, s) \simeq \mathbf{h}^{T}(x) K_{2} \mathbf{h}(s), \\
& f(x) \simeq F^{T} \mathbf{h}(x),
\end{aligned}
$$

where $K_{1}, K_{2}$ are known $n m \times n m$-matrices and $F$ is an $n m$-vector.

After substituting the approximate Equations 14, 15 and 16 in Equation 1, we get

$$
\begin{aligned}
& U^{T} \mathbf{h}(x) \simeq F^{T} \mathbf{h}(x)+\lambda_{1} \mathbf{h}^{T}(x) K_{1} \int_{0}^{x} \mathbf{h}(s) \psi_{1}\left(s, U^{T} \mathbf{h}(s)\right) d s \\
& +\lambda_{2} \mathbf{h}^{T}(x) K_{2} \int_{0}^{1} \mathbf{h}(s) \psi_{2}\left(s, U^{T} \mathbf{h}(s)\right) d s .
\end{aligned}
$$

Functions $\quad \psi_{1}\left(s, U^{T} \mathbf{h}(s)\right)=\left(U^{T} \mathbf{h}(s)\right)^{\alpha} \quad$ and $\psi_{2}\left(s, U^{T} \mathbf{h}(s)\right)=\left(U^{T} \mathbf{h}(s)\right)^{\beta}$ are known which can be expanded into the hybrid functions as

$$
(u(s))^{\alpha} \simeq U_{\alpha}^{T} \mathbf{h}(s),(u(s))^{\beta} \simeq U_{\beta}^{T} \mathbf{h}(s) .
$$

where $U_{\alpha}, U_{\beta}$ are $n m$-vectors whose elements are nonlinear combination of the elements of the vector $U$ and are produced as follows:

From Equations 13 and 14, we have

$$
\begin{gathered}
(u(x))^{2} \simeq\left(U^{T} \mathbf{h}(x)\right)\left(U^{T} \mathbf{h}(x)\right)=U^{T} \mathbf{h}(x) \mathbf{h}^{T}(x) U \\
=U^{T} \tilde{U} \mathbf{h}(x)=U_{2} \mathbf{h}(x),
\end{gathered}
$$

where the vector $U_{2}=U^{T} \tilde{U}$ is an $n m_{\text {_row vector, then }}$ for $(u(s))^{3}$ we get

$$
\begin{gathered}
(u(x))^{3} \simeq\left(U^{T} \mathbf{h}(x)\right)\left(U_{2} \mathbf{h}(x)\right)=U^{T} \mathbf{h}(x) \mathbf{h}^{T}(x) U_{2}^{T} \\
=U^{T} \tilde{U}_{2}^{T} \mathbf{h}(x)=U_{3} \mathbf{h}(x) .
\end{gathered}
$$

Therefore with this method we can approximate $(u(s))^{\alpha}$ and $(u(s))^{\beta}$ for arbitrary $\alpha$ and $\beta$. Suppose that this method holds for $\alpha-1$ where $(u(x))^{\alpha-1}=U_{\alpha-1} \mathbf{h}(x)$, we shall obtain it for $\alpha$ as follows: 


$$
\begin{gathered}
(u(x))^{\alpha}=u(x) u(x)^{\alpha-1} \simeq\left(U^{T} \mathbf{h}(x)\right)\left(U_{\alpha-1} \mathbf{h}(x)\right) \\
=U^{T} \mathbf{h}(x) \mathbf{h}^{T}(x) U_{\alpha-1}^{T} \\
=U^{T} \tilde{U}_{\alpha-1}^{T} \mathbf{h}(x)=U_{\alpha} \mathbf{h}(x),
\end{gathered}
$$

We have a similar relation for $\beta$. So, the components of $U_{\alpha}$ and $U_{\beta}$ can be computed in terms of components of unknown vector $U$.

Substituting Equation 18 in Equation 17 produces

$$
U \mathbf{h}(x) \simeq F \mathbf{h}(x)+\lambda \mathbf{h}^{T}(x) K \int_{0}^{x} \mathbf{h}(s) \mathbf{h}^{T}(s) U_{\alpha}\left(\delta+\lambda \mathbf{h}^{T}(x) K_{2} \int_{0}^{1} \mathbf{h}\left(s \mathbf{h}^{T}(s) U_{\beta} d .\right.\right.
$$

Note that by use of Equations 9 and 13 we have $\int_{0}^{x} \mathbf{h}(s) \mathbf{h}^{T}(s) U_{\alpha} d s=\int_{0}^{x} \tilde{U}_{\alpha} \mathbf{h}(s) d s=\tilde{U}_{\alpha} P \mathbf{h}(x)$, by this relation and Equation 10, we get

$$
U^{T} \mathbf{h}(x) \simeq F^{T} \mathbf{h}(x)+\lambda_{1} \mathbf{h}^{T}(x) K_{1} \tilde{U}_{\alpha} P \mathbf{h}(x)+\lambda_{2} \mathbf{h}^{T}(x)\left(K_{2} D U_{\beta}\right) .
$$

In order to find $U$ we collocate Equation 22 in $\mathrm{nm}$ nodal points of Newton-Cotes as,

$x_{p}=\frac{2 p-1}{2 n m}, \quad p=1,2, \ldots, n m$,

then we have the following system of nonlinear equations

$$
U^{T} \mathbf{h}\left(x_{p}\right) \simeq F^{T} \mathbf{h}\left(x_{p}\right)+\lambda_{1} \mathbf{h}^{T}\left(x_{p}\right) K_{1} \tilde{U}_{\alpha} P \mathbf{h}\left(x_{p}\right)+\lambda_{2} \mathbf{h}^{T}\left(x_{p}\right)\left(K_{2} D U_{\beta}\right), \quad p=1,2, . ., n m
$$

This nonlinear system of equations can be solved by Newton's method. We used the "Mathematica 7" software to solve this nonlinear system. After solving nonlinear system (Equation 24) we can achieve $U$, then we will have our unknown $u(x)$ as $U^{T} \mathbf{h}(x)$, that is the approximate solution of NV-FIE (1).

\section{CONVERGENCE ANALYSIS}

We assume $(C(J)\|\|)$ the Banach space of all continuous functions on $J=[0,1]$ with norm | $f(x)||=\max _{0 \leq x \leq 1}|f(x)|$ and the following conditions on $k_{1}, k_{2}$ and $\psi_{1}, \psi_{2}$ for Equation 1. We define $k_{x} \equiv k(x, s)$ for $x, s \in[0,1]$.

1. $\lim _{x \rightarrow}\left\|k_{x}-k_{\tau}\right\|=0, \tau \in[0,1]$.
2. $M_{1} \equiv \operatorname{sup~} 0 \leq x, s \leq 1\left|k_{1}(x, s)\right|<\infty, M_{2} \equiv \sup _{0 \leq x, s \leq 1}\left|k_{2}(x, s)\right|<\infty$.

3. $\psi_{1}(s, x)$ and $\psi_{2}(s, x)$ are continuous in $s \in[0,1]$ and Lipschitz continuous in $x \in R$, that is, there exist constants $C_{1}, C_{2}>0$ for which

$\left|\psi_{1}\left(s, x_{1}\right)-\psi_{1}\left(s, x_{2}\right)\right| \leq C_{1}\left|x_{1}-x_{2}\right| \quad$ forall $x_{1}, x_{2} \in R$, $\left|\psi_{2}\left(s, x_{1}\right)-\psi_{2}\left(s, x_{2}\right)\right| \leq C_{2}\left|x_{1}-x_{2}\right| \quad$ forall $x_{1}, x_{2} \in R$.

\section{Theorem 1}

The solution of nonlinear Volterra-Fredholm integral equation by hybrid functions converges if $0<\gamma<1$.

Proof: (Maleknejad et al., 2010)ㅁ.

\section{NUMERICAL EXAMPLES}

Here, we implemented the proposed method on 3 different examples. The results achieved by a proper value for $m$ and different values for $n$. All the results are compared with some existing method results. As in Tables 1 to 5, the error tends to zero when $n$ becomes greater and in analogy to another methods' results the proposed method have better answers in lower $n$. Although, we do not claim that this method shows superiority over the other methods from the viewpoint of accuracy, but we can say this method is more practical, quite accurate with lower calculation. The matrices $P$ and $D$ are sparse, hence are much faster than other functions' operational matrices and they reduce the CPU time and at the same time keeping the accuracy of the solution. In our examples we get the results by $m=8$ and $n=2,4,8,16$. In all of them we compared our answers with some existing methods. We consider the $L^{2}$-norm of errors for Examples 1 to 3 which are shown in Tables 4 and 5 by

$E_{2}=\left(\int_{0}^{1}\left(u(x)-u_{n m}(x)\right)^{2} d x\right)^{\frac{1}{2}}$

For implementation of proposed method we used Mathematica 7

\section{Example 1}

Consider the nonlinear Volterra-Fredholm equation given by

$u(x)=\frac{1}{30} x^{6}+\frac{1}{3} x^{4}-x^{2}+\frac{5}{3} x-\frac{5}{4}+\int_{0}^{x}(x-s) \mu^{2}(s) d s+\int_{0}^{1}(x+s)(s) d b$, 
Table 1. Approximate and exact solutions for Example 1.

\begin{tabular}{|c|c|c|c|c|c|c|}
\hline$x$ & $\begin{array}{l}\text { Solution with } \\
\qquad n=2\end{array}$ & $\begin{array}{c}\text { Solution with } \\
\qquad n=4\end{array}$ & $\begin{array}{l}\text { Solution with } \\
\qquad n=8\end{array}$ & $\begin{array}{l}\text { Solution with } \\
\qquad n=16\end{array}$ & $\begin{array}{c}\text { Method } \\
\text { (Ordokhani, } \\
\text { 2006) with } \\
k=16\end{array}$ & Exact \\
\hline 0.0 & -2.004858 & -2.001333 & -2.000340 & -2.000085 & -1.995 & -2 \\
\hline 0.1 & -1.996105 & -1.991671 & -1.990420 & -1.990104 & -1.989 & -1.99 \\
\hline 0.2 & -1.967892 & -1.962063 & -1.960511 & -1.960125 & -1.965 & -1.96 \\
\hline 0.3 & -1.919818 & -1.912286 & -1.910599 & -1.910152 & -1.912 & -1.91 \\
\hline 0.4 & -1.851187 & -1.842838 & -1.840683 & -1.840174 & -1.841 & -1.84 \\
\hline 0.5 & -1.761040 & -1.753034 & -1.750775 & -1.750195 & -1.752 & -1.75 \\
\hline 0.6 & -1.650618 & -1.643166 & -1.640844 & -1.640208 & -1.643 & -1.64 \\
\hline 0.7 & -1.521064 & -1.513388 & -1.510834 & -1.510204 & -1.498 & -1.51 \\
\hline 0.8 & -1.371005 & -1.362753 & -1.360742 & -1.360192 & -1.359 & -1.36 \\
\hline 0.9 & -1.198995 & -1.192383 & -1.190576 & -1.190149 & -1.185 & -1.19 \\
\hline 1.0 & -1.003603 & -1.001267 & -1.000339 & -1.000086 & -0.994 & -1 \\
\hline
\end{tabular}

Table 2. Approximate and exact solutions for Example 2.

\begin{tabular}{ccccccc}
\hline$x$ & $\begin{array}{c}\text { Solution with } \\
n=2\end{array}$ & $\begin{array}{c}\text { Solution with } \\
n=4\end{array}$ & $\begin{array}{c}\text { Solution with } \\
n=8\end{array}$ & $\begin{array}{c}\text { Solution with } \\
n=16\end{array}$ & $\begin{array}{c}\text { Method (Sepehrian and } \\
\text { Razzaghi, 2005) with } \\
M=20\end{array}$ & $\begin{array}{c}\text { Exact } \\
\text { (n) }\end{array}$ \\
\hline 0.0 & 0.00000 & 0.00000 & 0.00000 & 0.00000 & 0.00000 & -0.09 \\
0.1 & -0.09007 & -0.09002 & -0.09000 & -0.09000 & -0.09000 & -0.16 \\
0.2 & -0.16032 & -0.16008 & -0.16002 & -0.16000 & -0.16000 & -0.21 \\
0.3 & -0.21066 & -0.21018 & -0.21005 & -0.21001 & -0.20999 & -0.24 \\
0.4 & -0.24094 & -0.24027 & -0.24005 & -0.24001 & -0.23999 & -0.25 \\
0.5 & -0.25111 & -0.25022 & -0.25005 & -0.25001 & -0.24999 & -0.24 \\
0.6 & -0.24127 & -0.24013 & -0.24004 & -0.24000 & -0.23999 & -0.21 \\
0.7 & -0.21167 & -0.21025 & -0.21002 & -0.21001 & -0.20999 & -0.16 \\
0.8 & -0.16252 & -0.16047 & -0.16008 & -0.16002 & -0.15998 & -0.099 \\
0.9 & -0.09377 & -0.09075 & -0.09020 & -0.09004 & -0.099 & 0 \\
1.0 & -0.00503 & -0.00113 & -0.00027 & -0.00006 & 0.00003 & - \\
\hline
\end{tabular}

with the exact solution $u(x)=x^{2}-2$ [13]. The comparison among the hybrid solutions beside the exact solutions are shown in Table 1.

\section{Example 2}

Consider the nonlinear Volterra integral equation considered in (Sepehrian and Razzaghi, 2005)

$u(x)=\frac{15}{56} x^{8}+\frac{13}{14} x^{7} \frac{11}{10} x^{6}+\frac{9}{20} x^{5}+x^{2}-x+\int_{0}^{x}(x+s) u^{3}(s) d s$,

with the exact solution $u(x)=x^{2}-x$. The comparison among the hybrid solutions with $m=8$ and $n=2, n=4, n=8$ and $n=16$ beside the exact solutions are shown in Table 2.

\section{Example 3}

Consider the nonlinear Fredholm integral equation given in (Babolianet al., 2008) by

$u(x)=x^{2}-\frac{1}{12}+\frac{1}{2} \int_{0}^{1} s u^{2}(s) d s$,

with the exact solution $u(x)=x^{2}$. The comparison among the hybrid solutions beside the exact solutions are 
Table 3. Approximate and exact solutions for Example 3.

\begin{tabular}{ccccccc}
\hline$x$ & Solution with & Solution with & Solution with & Solution with & $\begin{array}{c}\text { Method (Babolian, 2008) } \\
\text { Exact }\end{array}$ \\
\hline 0.0 & $n=2$ & $n=4$ & $n=8$ & $n=16$ & 0.000054 & with $m=64$ \\
0.1 & 0.020629 & 0.005630 & 0.001436 & 0.000361 & 0.010308 & 0.01 \\
0.2 & 0.060629 & 0.045630 & 0.041436 & 0.040361 & 0.038140 & 0.092828 \\
0.3 & 0.110629 & 0.095630 & 0.091436 & 0.090361 & 0.158746 & 0.09 \\
0.4 & 0.180629 & 0.165630 & 0.161436 & 0.160361 & 0.257867 & 0.25 \\
0.5 & 0.270629 & 0.255630 & 0.251436 & 0.250361 & 0.361871 & 0.36 \\
0.6 & 0.380629 & 0.365630 & 0.361436 & 0.360361 & 0.483453 & 0.49 \\
0.7 & 0.510629 & 0.495630 & 0.491436 & 0.490361 & 0.647515 & 0.64 \\
0.8 & 0.660629 & 0.645630 & 0.641436 & 0.640361 & 0.807183 & - \\
0.9 & 0.830629 & 0.815630 & 0.811436 & 0.810361 & 1 \\
1.0 & 1.020629 & 1.005630 & 1.001436 & 1.000361 & 0.81 \\
\hline
\end{tabular}

Table 4. Errors $E_{2}$ for Examples 1.

\begin{tabular}{cccc}
\hline$m$ & Method in [10] & $n$ & present method $m=8$ \\
\hline 4 & 0.011410886610 & 2 & 0.009508596380 \\
8 & 0.002852721653 & 4 & 0.002578626395 \\
16 & 0.000713180414 & 8 & 0.000656705013 \\
32 & 0.003779018306 & 16 & 0.000164921678 \\
\hline
\end{tabular}

Table 5. Errors $E_{2}$ for Examples 2 to 3.

\begin{tabular}{ccc}
\hline$n$ & Example 2 & Example 3 \\
\hline 2 & 0.001986101366 & 0.020629858826 \\
4 & 0.000407433748 & 0.005630249634 \\
8 & 0.000098383419 & 0.001363230304 \\
16 & 0.000024414251 & 0.000361078218 \\
\hline
\end{tabular}

shown in Table 3. Our achieved errors for Example 1 is comparable with exhibited errors (Maleknejad et al., 2010), an example is shown in Table 4. As obvious in present method with lower $n$ gets better results.

\section{Conclusion}

This work presents a numerical approach for solving NVFIEs based on the hybrid Legendre polynomials and Block-Pulse functions. These hybrid functions operational matrices of integration $D$, operational matrix $P$, product matrix $H$ and coefficient matrix $\tilde{C}$ have been created to convert NV-FIEs to an algebraic equation and then by collocating this equation in Newton-cuts nodes, a nonlinear system of equations that can be solved by Newton method, was produced. Illustrative examples are given to demonstrate the validity and applicability of proposed method. Our compared results show that this method works better and faster than some existing methods.

\section{Acknowledgment}

The authors would like to thank Islamic Azad University of Birjand Branch for partially financially supporting this research and providing facilities and encouraging this work. 


\section{REFERENCES}

Abdou MA (2003). On asymptotic methods for Fredholm-Volterra integral equation of the second kind in contact problems. J. Comput. Appl. Math., 154: 431-446.

Babolian E, Masouri Z, Hatamzadeh-Varmazyar S (2008). New direct method to solve nonlinear Volterra-Fredholm Integral and Integro-Differential equations using operational matrix with Block-Pulse functions. Prog. Electromagn. Res. B., 8: 59-76.

Bloom F (1980). Asymptotic bounds for solutions to a system of damped integro-differential equations of electromagnetic theory. J. Math. Anal. Appl., 73: 524-542.

Chang RY, Wang ML (1983). Shifted Legendre direct method for variational problems. J. Opim. Theory Appl., 39: 299-307.

Hsiao CH (2009). Hybrid function method for solving Fredholm and Volterra integral equations of the second kind. J. Comput. Appl. Math., 230: 59-68.

Jaswon MA, Symm GT (1977). Integral Equation Methods in Potential Theory and Elastostatics. London: Academic Press. 275(23).

Jiang S, Rokhlin V (2004). Second kind integral equations for the calssical potential theory on open surface II. J. Comput. Phys., 195: 1-16

Maleknejad K, Almasih H, Roodaki M (2010). Triangular functions method for the solution of nonlinear Volterra-Fredholm integral equations. Commun. Nonlinear Sci. Numer. Simul., 15: 32933298.

Maleknejad K, Hashemizadeh E, Basirat B (2011). Computational method based on Bernstein operational matrices for nonlinear Volterra-Fredholm-Hammerstein integral equations. Commun. Nonlinear. Sci. Numer. Simulat. In Press. doi:10.1016/j.cnsns. 04.023.

Marzban HR, Razzaghi M (2003). Hybrid functions approach for linearly constrained quadratic optimal control problems. Appl. Math. Model, 27: 471-485.

Ordokhani Y (2006). Solution of nonlinear Volterra-FredholmHammerstein integral equations via rationlized Haar functions. Appl. Math. Comput., 180: 436-443.

Ordokhani Y, Razzaghi M (2008). Solution of nonlinear VolterraFredholm-Hammerstein integral equations via a collocation method and rationalized Haar functions. Appl. Math. Lett., 21: 49 .
Schiavane P, Constanda C, Mioduchowski A (2002). Integral Methods in Science and Engineering. Birkhauser, Boston, p. 264 ISBN 0-8176-4213-7.

Schiavane P, Constanda C, Mioduchowski A (2002). Integral Methods in Science and Engineering. Birkhauser, Boston, pp. 250-258.

Semetanian BJ (2007). On an integral equation for axially symmetric problem in the case of an elastic body containing an inclusion. J. Comp. Appl. Math., 200: 12-20.

Sepehrian B, Razzaghi M (2005). Solution of nonlinear VolterraHammerstein integral equations via single-term walsh series method. Math. Probl. Eng., 5: 547-554.

Voitovich NN, Reshnyak OO (1999). Solutions of nonlinear integral equation of synthesis of the linear antenna arrays. BSUAE J. App. Electr., 2(1): 43-52.

Yalcinbas S (2002). Taylor polynomial solution of nonlinear Volterra-Fredholm integral equations. Appl. Math. Comput., 127: 195-206.

Yousefi S, Razzaghi M (2005). Legendre wavelets method for the nonlinear Volterra-Fredholm integral equations. Math. Comp. Simul., 70: 1-8. 